\title{
Impact of presymptomatic genetic testing on young adults: a systematic review
}

\author{
Lea Godino ${ }^{\star, 1,2}$, Daniela Turchetti ${ }^{1}$, Leigh Jackson ${ }^{2}$, Catherine Hennessy ${ }^{2}$ and Heather Skirton ${ }^{2}$
}

Presymptomatic and predictive genetic testing should involve a considered choice, which is particularly true when testing is undertaken in early adulthood. Young adults are at a key life stage as they may be developing a career, forming partnerships and potentially becoming parents: presymptomatic testing may affect many facets of their future lives. The aim of this integrative systematic review was to assess factors that influence young adults' or adolescents' choices to have a presymptomatic genetic test and the emotional impact of those choices. Peer-reviewed papers published between January 1993 and December 2014 were searched using eight databases. Of 3373 studies identified, 29 were reviewed in full text: 11 met the inclusion criteria. Thematic analysis was used to identify five major themes: period befeore testing, experience of genetic counselling, parental involvement in decision-making, impact of test result communication, and living with genetic risk. Many participants grew up with little or no information concerning their genetic risk. The experience of genetic counselling was either reported as an opportunity for discussing problems or associated with feelings of disempowerment. Emotional outcomes of disclosure did not directly correlate with test results: some mutation carriers were relieved to know their status, however, the knowledge they may have passed on the mutation to their children was a common concern. Parents appeared to have exerted pressure on their children during the decision-making process about testing and risk reduction surgery. Health professionals should take into account all these issues to effectively assist young adults in making decisions about presymptomatic genetic testing. European Journal of Human Genetics (2016) 24, 496-503; doi:10.1038/ejhg.2015.153; published online 15 July 2015

\section{INTRODUCTION}

Presymptomatic and predictive genetic testing are available for a number of heritable genetic disorders including hereditary cancer syndromes, inherited cardiac conditions and neurodegenerative genetic disorders. ${ }^{1}$ The terms 'presymptomatic' and 'predictive' genetic testing refer to the possibility of detecting a genetic mutation that causes a particular condition before the presentation of symptoms. The first term generally refers to those diseases in which a positive test result will inevitably lead to the development of the disease later in life (eg, Huntington disease (HD)); the second term refers to a broader range of diseases in which the risk for a disorder is increased but without necessarily implying any degree of certainty (eg, hereditary breast and ovarian cancer (HBOC)). However, these terms are often used in a broadly interchangeable manner. A substantial difference is that cancer disorders can be monitored through a surveillance protocol or prevented by surgical intervention, while no prevention is currently available for diseases such as $\mathrm{HD}$ or cerebellar ataxia. Therefore, the choice to undergo a presymptomatic test for disorders with incomplete penetrance and where there are preventive measures could have a highly different psychological and social impact when compared with testing for disorders with complete penetrance and no preventive options, particularly in young adults. In this review, the term 'presymptomatic' will be used to indicate both predictive and presymptomatic tests, but the different impact will be considered whenever appropriate.

A presymptomatic diagnosis of a serious genetic illness can have a profound impact on the person and family and should be managed using an individualized counselling process. ${ }^{1}$ Presymptomatic genetic testing of minors (under the age of 18 years) is not usually recommended unless effective clinical actions are available. ${ }^{2-4}$ Generally, there are three key arguments against presymptomatic genetic testing in adolescents or young people: that it (1) fails to respect the future autonomy of the young person; (2) breaches confidentiality; and (3) may cause psychosocial harms. ${ }^{5}$

The age at which young people should undergo presymptomatic genetic testing for adult-onset disorder is a matter of debate. ${ }^{2-5}$ Key challenges have to be faced during the transition from adolescence to adulthood, such as marriage, finishing education, beginning full-time employment and becoming a parent, and the impact of testing may affect, and be affected by each of these events. In the light of the above-mentioned issues, it would be appropriate to ask what health information and counselling young adults need to make prudent decisions about genetic testing. The purpose of this systematic review was therefore to systematically identify and analyse factors influencing young adults' or adolescents' choices to have a presymptomatic test and the emotional impact of those choices.

\section{MATERIALS AND METHODS \\ Design}

A systematic review is a method of amassing, assessing and synthesizing a body of evidence on a particular topic. ${ }^{6}$ This systematic review was conducted in accordance with the Centre for Reviews and Dissemination methods for undertaking reviews in health care $^{6}$ with the aim of assessing which factors influence young adults' or adolescents' choices to have a presymptomatic test for a genetic condition and the emotional impact of those choices.

${ }^{1}$ Unit of Medical Genetics, Department of Medical and Surgical Sciences, Policlinico Sant'Orsola-Malpighi, University of Bologna, Bologna, Italy; ${ }^{2}$ School of Nursing and Midwifery, Faculty of Health and Human Sciences, Plymouth University, Plymouth, UK

*Correspondence: Dr L Godino, U.O. Genetica Medica, Dipartimento di Scienze Mediche e Chirurgiche, Policlinico Sant'Orsola-Malpighi, Padiglione 11, via Massarenti, 9 , 40138 Bologna, Italy. Tel: +39 51 2088425; Fax: +39 51 2088416; E-mail: lea.godino@students.plymouth.ac.uk

Received 30 January 2015; revised 29 May 2015; accepted 3 June 2015; published online 15 July 2015 


\section{Search strategy}

Eight databases used for indexing medical and psychosocial research (Embase, Medline, PsychoInfo, Pubmed, SocIndex, The Cochrane Library, Cumulative Index of Nursing Allied Health Literature (CINAHL) and Web of Science) were searched for papers published between January 1993 and December 2014. We chose to start at 1993 because presymptomatic testing based on mutation analysis (ie, not based on linkage) became available for HD that year (Harper et $\left.a l^{7}\right)$ and this was a landmark in presymptomatic testing for adult onset conditions. As the first authors are bilingual, papers published in either English or Italian were eligible (there were no papers identified that were written in Italian). The literature search employed variations and Boolean connectors of the key terms. An exploratory search with the terms 'genet*' ${ }^{*}$ ' 'predict $^{*}$ test ${ }^{*}$

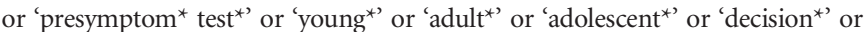
'choic*' or 'communicat" or 'psycho*' resulted in 976 studies, which failed to include some papers already known on this theme, therefore a further search was conducted by using the key terms: 'adolescent*' or 'young*' or 'BRCA' or 'APC' or 'Lynch' or 'Huntington' or 'genetic ${ }^{\star}$ test ${ }^{*}$. Targeted internet searching using Google Scholar and reference lists of relevant papers were also examined for any additional studies of interest.

\section{Inclusion and exclusion criteria}

The criteria for inclusion in this systematic review were papers: (a) published in English or Italian; (b) published in peer-reviewed journals between 1993 and 2014 and reporting original research (using any methods); (c) where the study sample explicitly included young adults or adolescents (14-30 years); (d) focused on presymptomatic or predictive testing in young people; (e) focused on the factors influencing young adults' or adolescents' choices to have a presymptomatic or predictive test and the emotional impact of those choices. Papers were excluded from the review if they were (a) guidelines for testing; (b) educational or opinion papers; (c) focused on perceptions and attitudes of college students/young adults who were not at known risk of a specific adult-onset genetic condition.

\section{Selection of studies}

Three review authors (LG, LJ and DT) independently screened the titles and abstracts of articles identified in the first search against the inclusion criteria and decided which papers should be retrieved. Articles were rejected at this stage if the title or abstract did not focus on our topic, was not in English or Italian, or was not original research. We reviewed selection decisions and resolved disagreements by consultation with a third review author (HS).

\section{Search outcome}

The Preferred Reporting Items for Systematic Reviews and Meta-Analyses $(\text { PRISMA })^{8}$ flowchart showing the consecutive methodological steps of this systematic review is displayed in Figure 1. The search of eight databases initially produced 976 potential papers. With the second search, 2397 papers were found. On the total of 3373, 755 were duplicates, leaving 2618 for examination. Following review of the title and abstract, 29 papers were assessed as potentially relevant. These papers were read in detail by the authors. Eleven papers were included in the review. The main characteristics of the included studies are described in Table 1.

\section{Quality appraisal}

All papers considered for inclusion in the review were subjected to independent analysis by two authors (LG and HS) using standard quality assessment criteria for evaluating original research papers from a variety of fields. ${ }^{9}$ This evaluation method allows the systematic evaluation of both quantitative and qualitative original research and across a broad range of study designs. Specific aspects of the paper relating to methodology and reporting of results are assessed and assigned 0 points (not addressed), 1 point (partially addressed) or 2 points (satisfactorily addressed). A summary percentage score was calculated by dividing total score summed across all applicable items by the highest possible score total after excluding non-applicable items. ${ }^{9}$ Any disagreements about scoring of papers were discussed. Kmet et al ${ }^{9}$ do not enforce a minimum score for inclusion in a review, although they suggest $60 \%$ as a reasonable cutoff point. In the present review two papers scored $50 \%$, but it was decided to

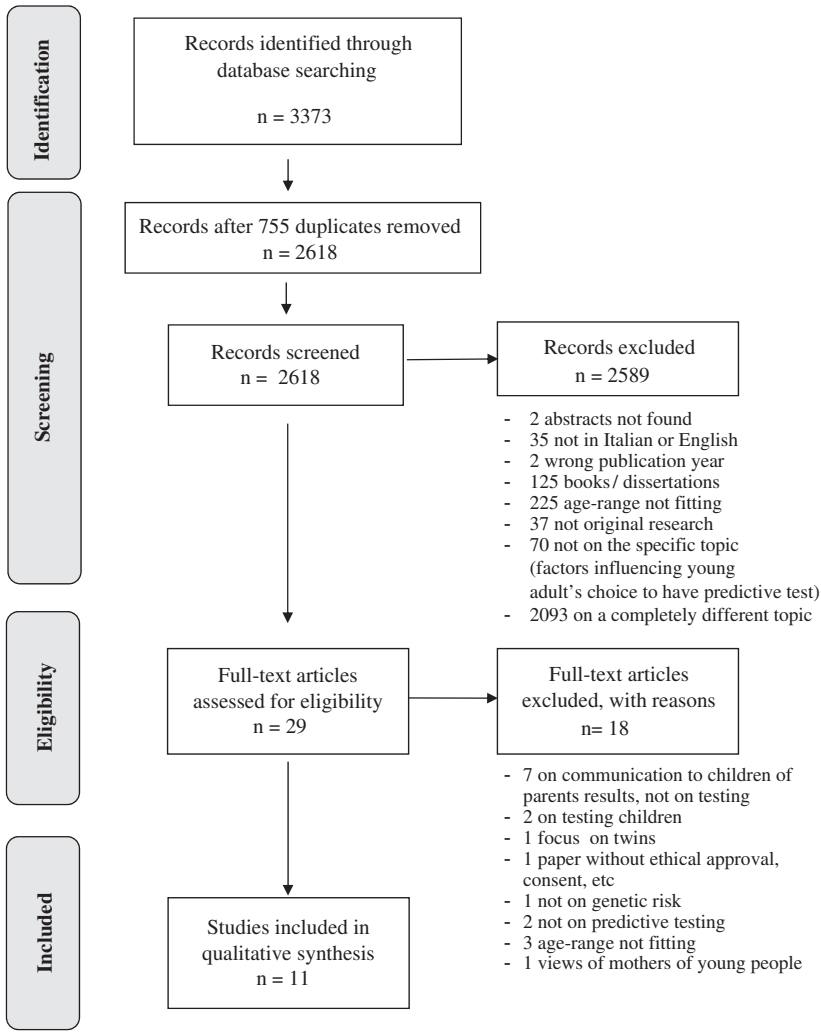

Figure 1 PRISMA flowchart of study selection. This PRISMA flowchart demonstrates the process of identifying and screening relevant studies. The screening process identified 11 studies from an initial pool of 3373 as being relevant to the current review.

include them, since they contributed relevant information that was not included in the other papers (see Table 1). Thus, all papers contributed to the synthesis and development of themes.

\section{Data abstraction and analysis}

It was not possible to undertake a meta-analysis of the data due to the heterogeneity of the methods and samples. We therefore prepared a narrative description of the findings ${ }^{6}$ using thematic analysis based on the methods described by Braun and Clarke, ${ }^{10}$ in order to employ a clear, replicable and transparent methodology. The thematic analysis was confirmed by three authors (LG, DT and HS).

\section{RESULTS}

\section{Characteristics of included studies}

In total, the systematic review included 11 qualitative studies. Methods adopted by the authors were interpretative phenomenological analysis, ${ }^{11}$ thematic analysis, ${ }^{12-16}$ a combination of interpretative content analysis and thematic analysis, ${ }^{17}$ or grounded theory. ${ }^{17-21}$ Patenaude et $a^{16}$ also included a quantitative analysis of their data.

All the included studies were published between 2007 and 2014 and were focused on few specific heritable disorders, namely autosomal dominant cerebellar ataxia, ${ }^{15}$ familial adenomatous polyposis (FAP) ${ }^{13,17}$ familial cardiomyopathy, ${ }^{11}$ HBOC, ${ }^{11,14-16,18-21}$ hereditary diffuse gastric cancer, ${ }^{15} \mathrm{HD}^{2,12,15,17}$ and Lynch syndrome. ${ }^{15}$ Samples included participants within an age range of 12-39 years, thus including, but not limited to, the age range of 14-30 years identified by the authors as the focus of the search. Eligible studies were conducted in Australia, ${ }^{12,13,15,17}$ Canada, ${ }^{14,18,19}$ United Kingdom, ${ }^{11}$ and the United States. ${ }^{16,18-20}$ 


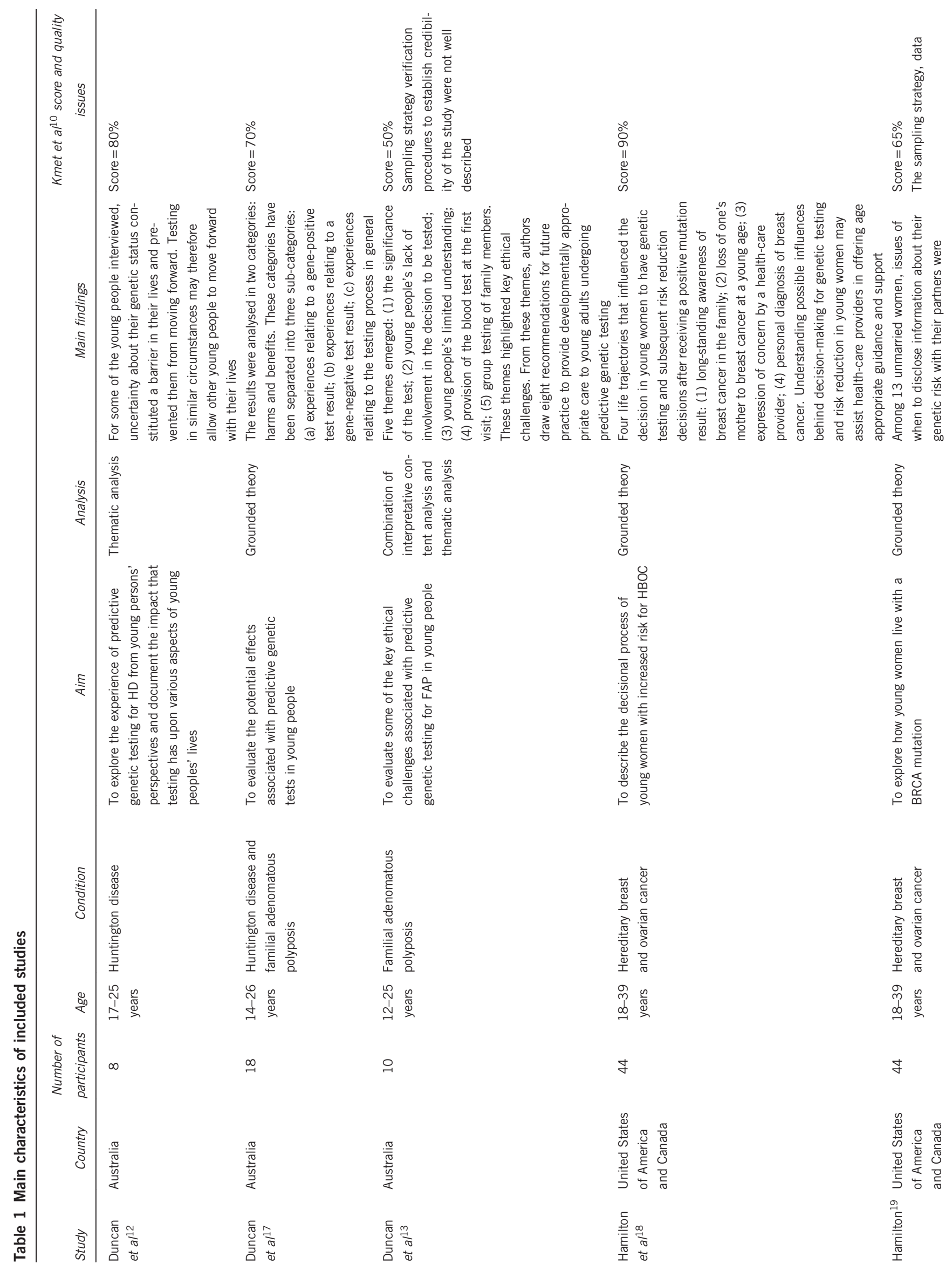




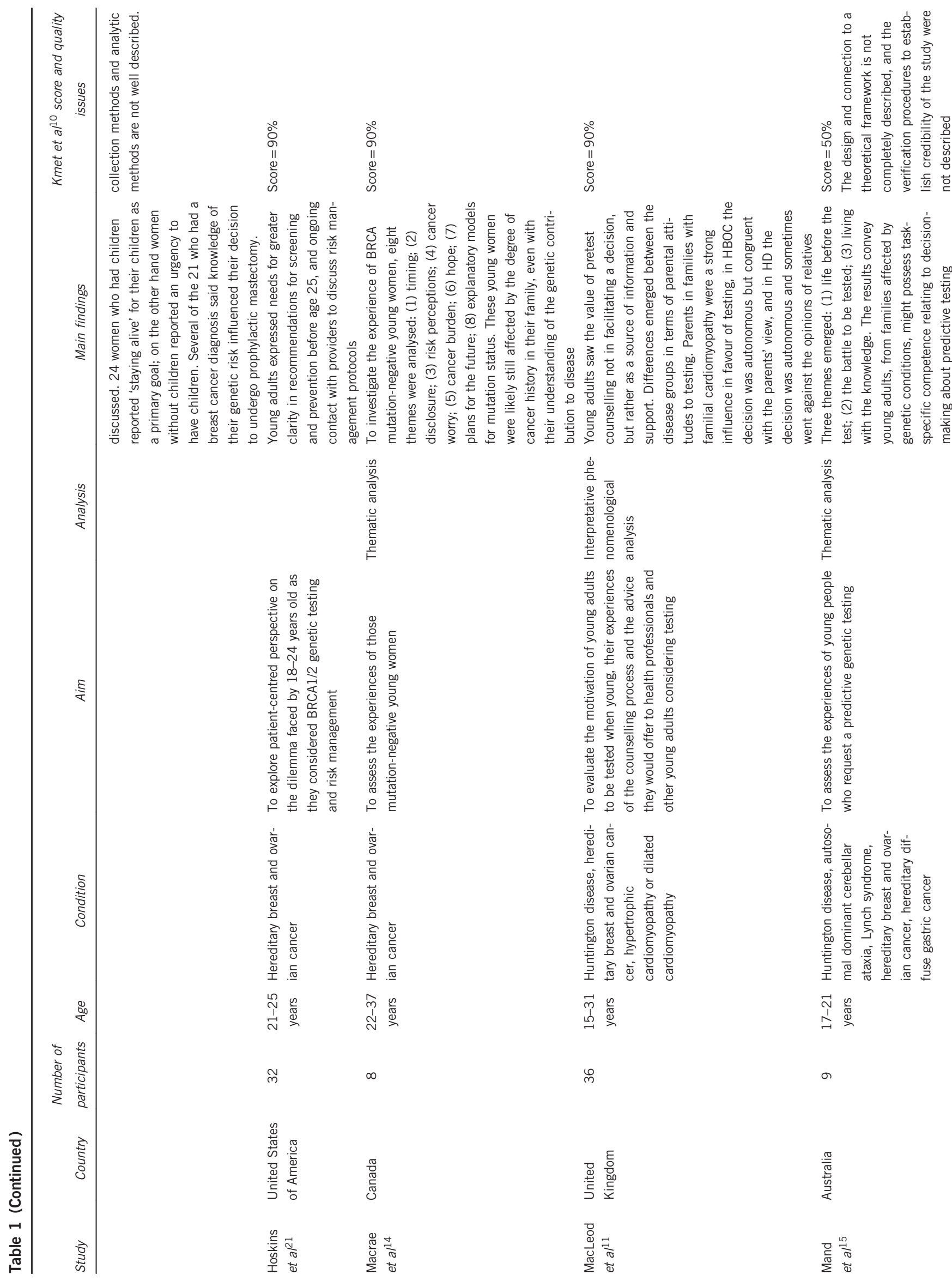


Findings of the analysis

Issues emerging from young people interviews included family and partner relationships, plans for the future, emotional state and the general approach to life.

Five major themes were identified: the period before testing, the experience of genetic counselling, involvement of parents in decisionmaking, impact of personal test result communication and living with genetic risk.

The period before testing. Many participants reported having grown up without awareness or with misinformation about the genetic disease running in their family or its inheritance mode: ${ }^{12,16,21}$ they also lacked information about the appropriate age for testing. 12,16

Two sets of authors reported that the first communication about the genetic risk was made by parents. ${ }^{14,16}$ None of the participants was younger than 12 years of age when informed, about half experienced disclosure before they were 18 years old and half between 18 and 21 years old. Many participants stated that the disclosure was made during an occasional encounter and in a casual moment (ie, while driving) or by telephone. ${ }^{16}$ Almost all daughters were informed of their mother's test result in a private conversation with their mother, and it was rare for both parents to participate in the disclosure. ${ }^{14,16}$ In some studies, participants expressed a preference toward being informed by both parents, although they knew that the information given by parents was limited and sought genetic counselling almost immediately after disclosure. ${ }^{14}$ In other cases, once aware of the family genetic disorder, those who did not understand what it really meant sought information online or in professional journals, ${ }^{16}$ while those who were more conscious of own risk (or potential risk) arranged the first counselling session to have their blood test. ${ }^{18}$

However, interviewees described the disclosure of a positive parental test result as the most important information of their lives, ${ }^{15}$ reporting concerns about their mother's health ${ }^{15,16}$ and, only secondarily, their own. ${ }^{15}$ In the quantitative sub-study by Patenaude et al, ${ }^{16}$ one-third of the daughters of BRCA1/2 mutation carriers reported normal levels of general distress but high cancer-related distress, which was not significantly different from distress levels of women with known BRCA1/2 mutations.

Some participants reported that at the time they were told of their risk, the implications for themselves seemed distant, but now, as young adults, the fear of developing the adult-onset disease recurring in their family had increased. ${ }^{16}$ Conversely, others felt that early disclosure of the family disease gave them the time to digest the information. ${ }^{14}$ However, the knowledge of being at risk of a disorder such as HD for some participants involved engagement in risk behaviours such as drugs use, trouble with the police or difficulty at school. ${ }^{12}$

When approaching the decision about testing, in the study by MacLeod et $a l^{11}$ most of the participants did not understand that having a presymptomatic test was a choice, but rather something they felt obliged to undergo in order to obtain information about themselves and to remove uncertainty. For example, a young woman said 'I knew, I had to' ( $\mathrm{p}$ 397). ${ }^{11}$ By contrast, those who perceived there was a choice prepared themselves for the result; some prepared themselves for the worst possible outcome because then they would not be surprised by bad news, ${ }^{11,12,15}$ while other participants were scared that receiving the test result would be devastating. ${ }^{16}$ Some study participants expected to test positive because of identification with a gene-positive family member. ${ }^{11,14,15}$

Choosing to undergo genetic testing constituted a major life event, ${ }^{13}$ so important that participants reported it had a significant impact on their outlook and sense of self. ${ }^{15,17}$ For example, a young woman said 
'that was the day the clock stopped; that was the day the uncertainty began' (p 644). ${ }^{15}$ Nevertheless, while Mand et al ${ }^{15}$ and MacLeod et al ${ }^{11}$ report that no interviewees expressed regret regarding the decision to undertake testing, the timing for testing emerged as important because of potential interference with schooling: one young woman tested during her final year, said that looking back, she wished she had been tested at a different time of her life. ${ }^{17}$ Another factor relevant to the timing of testing was childbearing planning, ${ }^{11,14}$ with participants split on the issue of undergoing presymptomatic testing before versus after having children. ${ }^{14}$

The experience of genetic counselling. Undergoing genetic counselling for the young adults studied by Duncan et $a l^{17}$ was reported to have helped discussion of problems, for example a young woman who was mutation positive for HD said that the counsellor helped her with every type of problem in her life. Even when the genetic counselling was a source of information and support, it did not appear to facilitate the decision to be tested. ${ }^{11}$ However, Duncan et al ${ }^{13}$ showed that when counselled and tested at the same time as their siblings, participants felt this had limited the individual attention and support during the counselling process.

Some negative feelings were reported about counsellors, ${ }^{15}$ such as the perception of not being understood and the feeling that the counsellor was the person with the power over the testing decision. Some participants were disappointed to hear that the counsellor believed they were not ready to deal with the psychological consequences of the genetic test and that they needed to take time to reach an autonomous decision. The need to wait for genetic testing increased the feeling of disempowerment raised by uncertainty: a girl said ' $I$ wanted the maybe to become yes or no; I was over maybe' ( $\mathrm{p} \mathrm{645).}{ }^{15}$ Others focused their attention on the procedure, instead of the meaning of testing, with the fear of the needle overshadowing the purpose of the counselling. ${ }^{13,17}$

A consequence of discussion during pre-test counselling was that most young adults had shared their test result with only close friends and family, because they felt that other people would not understand the complexities of the process from decision-making to the result. ${ }^{11}$

Involvement of parents in decision-making. Although theoretically an autonomous choice to undergo presymptomatic testing is a fundamental requirement of the process of genetic counselling, some parents were reported to exert pressure on their young adult children. ${ }^{11-13,17,18,20}$ In addition, Hoskins et al ${ }^{21}$ reported that a young woman underwent genetic testing because of her gynaecologist's suggestion. As a consequence of parental pressure, interviewees conveyed feelings of disempowerment and lack of control and declared that they underwent genetic testing because of pressure from family members or 'for' a parent, which also raised the ethical problem of respecting young adults' developing autonomy. ${ }^{12,13,17,18,20}$ A young boy said 'I was 12 when I was told that I had to have the test, I didn't want to have it, but then I sort of had to' ( $\mathrm{p} \mathrm{30)} ;{ }^{13}$ a girl said that her parents did not ask her if she wanted to do, they just said 'you know, you have to go get a blood test' ( $\mathrm{p} 30){ }^{13}$

Older participants were more likely than younger ones to decide autonomously to have genetic testing, so much so that some described it as ' $a$ way to take control and not to be like their mothers' ( $\mathrm{p} \mathrm{152).}{ }^{19}$

Even when the decision-making was autonomous and pragmatic, still the family experience was important, especially when parents had developed cancer. ${ }^{18}$ Some of these young adults had lost a parent in their adolescence or earlier, so they grew up without a parent and with the knowledge that their parent's death was due to 'the gene' and that they may carry the same risk. In this way, the study conducted by Hamilton et $a l^{18}$ showed that participants both desired and feared genetic testing. Another key motivation was the perception that they were doing something to alter the course of a disease that had led to the death of affected relatives. ${ }^{11}$ A young woman said 'I just thought that you know if she (mum) would have had the opportunity to have the test, then things could have been a lot different' (p397). ${ }^{11}$

However, differences emerged in parental involvement in the decision-making process, based on the specific disease. ${ }^{11}$ Parents in families with familial cardiomyopathy had a strong influence in favour of testing; in HBOC the decision was autonomous but usually congruent with the parents' point of view, while in HD the decision was autonomous and sometimes went against the parents' opinion. ${ }^{11}$

Impact of personal test result communication. Once the test was undertaken, waiting for test result was associated with anxiety. ${ }^{17}$ At the time of the communication of the genetic test result, participants generally had to face the idea of disease. ${ }^{14}$ Usually at their age young adults do not think about disease; their attention is focused on plans for their future such as university and/or job plans. ${ }^{14}$ Nevertheless, none of the participants of the study by Mand et al 15 reported a catastrophic emotional response to their test result, but conflicting emotions of relief, happiness, guilt, fear and anger were generally reported. ${ }^{14}$ In more detail, authors described the emotional impact of both gene-negative and gene-positive test results. Surprisingly, positive and negative emotional outcomes were not correlated with test results: in any case interviewees thought that the best thing was to find out the result. ${ }^{17}$ Accordingly, participants described themselves as happy just to know their genetic status or as willing to begin enjoying life and to make behavioural changes. ${ }^{12,15}$ Specifically, a positive result led participants to feel able to move forward and to understand what was important (or not) in their lives. ${ }^{17}$ Although some participants stated their positive result induced a change of lifestyle, others showed no reaction to testing positive; this lack of reaction sometimes created uneasiness because the counsellors failed to understand the underlying feelings, which are well explained by a girl: 'I kept on the same direction I was already going' (p 646). ${ }^{15}$ In others, a gene-positive result created some negative emotions such as depression and anxiety, either in general or related to potential gossip by other people, ${ }^{15,17}$ connected with employment, ${ }^{17}$ related to the possibility of passing on the mutation to their future children ${ }^{15}$ or because of a different test result in other family members. ${ }^{11}$ A young boy said 'when I first found out I didn't want be too happy around them because it's still not the best of situations because my mum's still poorly with it [...], I still upset about my mum' (p 399); ${ }^{11}$ a girl said that she had been only thinking of herself during the decision-making process, but now, receiving a negative test result, she wondered ' $w$ hat does she (sister) feel about me now because I haven't got it and she has' (p 399). ${ }^{11}$ In addition, some interviewees described their shock at finding out that they had not inherited the family mutation: ${ }^{11,15}$ they had prepared themselves for something and then it just did not happen. ${ }^{11,12}$ This was particularly true for young adults receiving an HD result. ${ }^{11}$ One young man, in the study by Macrae et al, ${ }^{14}$ received a negative test result but clearly expressed the desire to have been mutation positive. Also, negative test results generated unexpected negative emotions in some participants, such as guilt and feeling distanced from family members. Moreover, some interviewees expressed the desire to receive additional screening regardless of their results, because of their familial cancer experience and residual cancer worry. ${ }^{14}$ Nevertheless, in other participants the negative test result was associated with feeling able to plan for the future. ${ }^{17}$ 


\section{Living with a genetic risk}

Although the authors of seven papers analysed risk management in terms of behaviour and attitudes, six of those papers were focused on BRCA1/2 carriers or daughters of BRCA1/2 carriers $^{11,14,16,18-21}$ and only one on familial cardiomyopathy and HD. ${ }^{11}$

Even though interviewees stated that having time before the beginning of surveillance gave them the opportunity to think about surveillance protocols or prophylactic surgery, ${ }^{14}$ younger participants were more likely to feel out of place in the health-care system and frustrated at their inability to access screening, so much so that some described themselves as 'paralyzed': one young woman underwent bilateral mastectomy at age 22, believing it was the only way to manage her cancer risk. ${ }^{21}$ Others expressed frustration at receiving inconsistent, inaccurate, ambiguous or incomplete recommendations by genetic counsellors or doctors, during the initial phase of their mutationpositive experience. ${ }^{21}$ They complained that each doctor explained only their own discipline-specific perspective and knowledge base.

Others wondered about when to share with a new partner their genetic risk or how early in a relationship to discuss having children ${ }^{11,16,19-21}$ or plans for prophylactic surgery. ${ }^{16,19,20}$

Some participants with children described the impact of knowing they may have passed on the mutation to their children in terms of feeling guilty, worried and, in some cases, leading to a decision to limit the number of children. However, none regretted the choice to have children $;^{19}$ they declared that they first considered the possibility of avoiding having children, but then realized that there were many options and over time there will hopefully be more. ${ }^{19}$ However, participants with children thought also about 'staying alive' related to their children ( $\mathrm{p} \mathrm{28})^{19}$ or of being not the next in the family. ${ }^{18}$ As a consequence of the wish to stay alive, young women were making the choice to have prophylactic surgery sooner rather than later. ${ }^{18,19}$ On the other hand, those opting for surveillance did not feel confident in surveillance protocols and reported of being anxious waiting for the next screening. ${ }^{18}$ A 35 -year-old woman said 'I admire women who can live with surveillance, but that was not for me' and she felt herself as being a 'ticking time bomb' (p 153). ${ }^{18}$

Werner-Lin et al, ${ }^{20}$ also, showed that some parents exerted pressure on their children to pursue risk reduction surgery, while other young adults erected a barrier, because of their young age, to address aspects of cancer risk, for example in terms of being too young for surveillance.

\section{DISCUSSION}

Although this systematic review focused on presymptomatic testing, one major issue emerging from the papers reviewed is when and how at-risk individuals are informed of their genetic risk. Although many participants grew up with no or scarce information concerning their potential genetic risk, communication generally occurred due to the parents' initiative and in a casual manner, several years before testing or clinical actions could be undertaken. ${ }^{12,16,21}$ This is in line with findings by Rew et al, ${ }^{22}$ which showed that the majority of children of BRCA mutation carriers learnt of their potential genetic risk of cancer many years before preventive interventions were recommended. Indeed, intra-familial communication is a highly complex process, especially when an inherited genetic condition is involved, thus it is understandable that parents face the dilemma of when, how and what to tell their children about it. ${ }^{23,24}$ On the other hand, appropriate communication of genetic risk information by parents to their children is highly desirable, since it has been shown to have longterm consequence in terms of informed reproductive decision-making and better family cohesion. ${ }^{25}$ To achieve this, health professionals may have a role in both supporting parents and young people, but their involvement in parents' decisions to communicate genetic risk to young family members was found to be limited in both our search and previous reports. ${ }^{22,26,27}$ Although this may be partly due to the parents' wish to undertake this task alone, it is reported that some parents desired health professionals to be available in a supporting role, but found that this support was limited. ${ }^{25,28}$ This evidence highlights the need for a comprehensive, longitudinal counselling process with appropriate timing and setting, which supports 'parent-to-offspring' risk communication first and young people's decision-making about presymptomatic testing and risk management afterwards. Accordingly, participants perceived that their lack of emotional experience at the time of testing had made it difficult for them to envisage the possible psychological impact of a test result. ${ }^{11}$ Furthermore, establishing a deeper and long-standing relationship with the counsellor may reduce the feelings of disempowerment reported by some study participants about the experience of genetic counselling. Such an approach would also help limit parents' pressure toward testing or risk-reducing surgery, which was a relevant issue in the studies reviewed. ${ }^{11-13,17,18,20}$

Concerning the impact of test results, overall, our findings do not support any substantial risk of adverse emotional outcome in mutation carriers, which is in agreement with previous findings. ${ }^{29}$ However, possible reactions to being tested per se should be explored before undertaking testing, instead of focusing only on the potential effects of specific test results.

Nevertheless, there is general concern that undergoing presymptomatic testing too early in life may increase the risk of unfavourable impact, and, therefore, the right age to undergo presymptomatic testing is still a matter of debate. ${ }^{30-32}$ In most of the papers analysed, the age at which participants had undergone genetic testing was not specified, ${ }^{13,14,16,18-20}$ whereas Duncan et al, ${ }^{17}$ who included in their analysis 10 individuals who were aged 10-17 years at the time of their genetic test for FAP, concluded that harms observed in younger persons were no different in nature from those described in adults. According to UK guidelines, people aged 16 or 17 are presumed to be capable of consenting to their own medical treatment, and, in specific cases, children under 16 years who have sufficient understanding and intelligence to enable them to fully understand what is involved in a proposed intervention will also have the capacity to consent to that intervention. ${ }^{33}$ Conversely, according to international guidelines, ${ }^{4}$ presymptomatic testing for adult-onset disorders is recommended to be made available to those aged 18 years and older, unless there it is in a child's best interest either in terms of immediate relevance for their health or of psychological or social benefits. Nevertheless, Richards ${ }^{31}$ argued that young persons who are considered as adults on the age-based criterion of 18 years are not all necessarily truly autonomous. She pinpointed that the most important aspect of the decision-making process is the recognition that the knowledge obtained from the test result is irreversible. There is not a specific age when a person is able to give autonomous consent, but it is important to consider psychological maturity ${ }^{31}$ that is cumulative with age, life experience and cognitive development. ${ }^{34}$ Therefore, future studies should aim at defining the optimal moment when to undergo presymptomatic genetic tests, not only on the basis of the age, but also considering psychosocial maturity. ${ }^{35}$ In any case, genetic health-care professionals, in the context of presymptomatic counselling, should support young adults to become aware of their own individual needs and capacities and of the fact that, sometimes, waiting to be tested may be helpful to better understand potential harms and benefits of testing.

In addition, although it is reasonable to hypothesize that undergoing testing at the right time reduces the risk of negative effects, it is 
important to consider the influence of the specific disease considered: the perception and experience of harms and benefits from the test result for a potentially treatable condition (such as BRCA and FAP, etc) may not be the same as for conditions for which there are no preventive treatment or cure (such as HD).

A potential limitation of this systematic review is that all the papers analysed are based on studies conducted in only four countries with similar British historical and cultural legacies, thus the findings may not generalize to other countries with different sociocultural backgrounds, supporting the need for further studies in other contexts. On the other hand, the papers analysed spanned across several diseases, while considering similar age ranges, thus providing a comprehensive overview of how young adults deal with genetic testing overall and according to the specific disease.

\section{CONFLICT OF INTEREST}

The authors declare no conflict of interest.

\section{ACKNOWLEDGEMENTS}

LG is supported by the Grant from Regione Emilia-Romagna 'Diagnostics advances in hereditary breast cancer (DIANE)' (PRUa1GR-2012-001).

1 Evans JP, Skrzynia C, Burke W: The complexities of predictive genetic testing. BMJ 2001; 322: 1052-1056.

2 MacLeod R, Beach A, Henriques S, Knopp J, Nelson K, Kerzin-Storrar L: Experiences of predictive testing in young people at risk of Huntington/'s disease, familial cardiomyopathy or hereditary breast and ovarian cancer. Eur J Hum Genet 2013; 22: 396-401.

3 Borry P, Goffin T, Nys H, Dierickx K: Predictive genetic testing in minors for adult-onset genetic diseases. Mt Sinai J Med 2008; 75: 287-296.

4 Borry P, Evers-Kiebooms G, Cornel MC, Clarke A, Dierickx K: Genetic testing in asymptomatic minors: background considerations towards ESHG recommendations. Eur J Hum Genet 2009; 17: 711-719.

5 Clarke A: The genetic testing of children. Working Party of the Clinical Genetics Society (UK). J Med Genet 1994; 31: 785.

6 CRD: Systematic Reviews: CRD's Guidance for Undertaking Reviews in Health Care. Centre for Reviews and Dissemination: University of York, York, UK, 2009.

7 Harper PS. A specific mutation for Huntington's disease. J Med Genet 1993; 30 975-977.

8 PRISMA Statement website http://www.prisma-statement.org/ (accessed 28 October 2014).

9 Kmet LM, Lee RC: Standard quality assessment criteria for evaluating primary research papers from avariety of fields. HTA Initiat 2004; 13: 1-28.

10 Braun V, Clarke V: Using thematic analysis in psychology. Qual Res Psychol 2008; 3: 77-101.

11 MacLeod R, Beach A, Henriques S, Knopp J, Nelson K, Kerzin-Storrar L: Experiences of predictive testing in young people at risk of Huntington's disease, familial cardiomyopathy or hereditary breast and ovarian cancer. Eur J Hum Genet EJHG 2014; 22: 396-401.

12 Duncan RE, Gillam L, Savulescu J, Williamson R, Rogers JG, Delatyckil MB: "Holding your breath": Interviews with young people who have undergone predictive genetic testing for Huntington disease. Am J Med Genet Part A 2007; 143A 1984-1989.
13 Duncan RE, Gillam L, Savulescu J, Williamson R, Rogers JG, Delatycki MB: The challenge of developmentally appropriate care: predictive genetic testing in young people for familial adenomatous polyposis. Fam Cancer 2010; 9: 27-35.

14 Macrae L, de Souza AN, Loiselle CG, Wong N: Experience of BRCA1/2 mutationnegative young women from families with hereditary breast and ovarian cancer: a qualitative study. Hered Cancer Clin Pract 2013; 11: 14.

15 Mand C, Gillam L, Duncan RE, Delatycki MB: "It was the missing piece": adolescent experiences of predictive genetic testing for adult-onset conditions. Genet Med 2013; 15: 643-649.

16 Patenaude AF, Tung N, Ryan PD, Ellisen LW, Hewitt L, Schneider KA et al: Young adult daughters of BRCA1/2 positive mothers: What do they know about hereditary cancer and how much do they worry? Psychooncology 2013; 22: 2024-2031.

17 Duncan RE, Gillam L, Savulescu J, Williamson R, Rogers JG, Delatycki MB: "You're one of us now": young people describe their experiences of predictive genetic testing for Huntington disease (HD) and familial adenomatous polyposis (FAP). Am J Med Genet C Semin Med Genet 2008; 148c: 47-55.

18 Hamilton R, Williams JK, Bowers BJ, Calzone K: Life trajectories, genetic testing, and risk reduction decisions in 18-39 year old women at risk for hereditary breast and ovarian cancer. J Genet Couns 2009; 18: 147-159.

19 Hamilton R: Being young, female, and BRCA positive. Am J Nurs 2012; 112: 26.

20 Werner-Lin A, Hoskins LM, Doyle MH, Greene MH: "Cancer doesn't have an age" genetic testing and cancer risk management in BRCA1/2 mutation-positive women aged 18-24. Heal (London, Engl 1997) 2012; 16: 636-654.

21 Hoskins LM, Werner-Lin A, Greene MH: In their own words: treating very young BRCA1/2 mutation-positive women with care and caution. PLoS One 2014; 9: e87696.

22 Rew L, Mackert M, Bonevac D: A systematic review of literature about the genetic testing of adolescents. J Spec Pediatr Nurs 2009; 14: 284-294.

23 Sobel SK, Cowan DB: Impact of genetic testing for Huntington disease on the family system. Am J Med Genet 2000; 90: 49-59.

24 Sobel S, Cowan CB: Ambiguous loss and disenfranchised grief: the impact of DNA predictive testing on the family as a system. Fam Process 2003; 42: 47-57.

25 Metcalfe A, Coad J, Plumridge GM, Gill P, Farndon P: Family communication between children and their parents about inherited genetic conditions: a meta-synthesis of the research. Eur J Hum Genet 2008; 16: 1193-1200.

26 Bradbury AR, Dignam JJ, Ibe CN, Auh SL, Hlubocky FJ, Cummings SA et al: How often do BRCA mutation carriers tell their young children of the family's risk for cancer? A study of parental disclosure of BRCA mutations to minors and young adults. J Clin Oncol 2007; 25: 3705-3711.

27 Werner-Lin A, Ratner R, Hoskins LM, Lieber C: A survey of genetic counselors about the needs of 18-25 year olds from families with hereditary breast and ovarian cancer syndrome. J Genet Couns 2015; 24: 78-87.

28 Gaff CL, Lynch E, Spencer L: Predictive testing of eighteen year olds: counseling challenges. J Genet Couns 2006; 15: 245-251.

29 Broadstock M, Michie S, Marteau T: Psychological consequences of predictive genetic testing: a systematic review. Eur J Hum Genet 2000; 8: 731-738.

30 Borry P, Stultiens L, Nys H, Cassiman J-J, Dierickx K: Presymptomatic and predictive genetic testing in minors: a systematic review of guidelines and position papers. Clin Genet 2006; 70: 374-381.

31 Richards FH: Predictive genetic testing of adolescents for Huntington disease: a question of autonomy and harm. Am J Med Genet Part A 2008; 146A: 2443-2446.

32 Duncan RE, Gillam L, Savulescu J, Williamson R, Rogers JG, Delatycki MB: Reply to Richards: "Predictive genetic testing of adolescents for Huntington disease: a question of autonomy and harm.". Am J Med Genet Part A 2008; 146A: 2447-2448.

33 Department of Health Reference Guide to Consent for Examination or Treatment, 2nd edn 2009; London: Department of Health.

34 Steinberg L, Cauffman E: Maturity of judgment in adolescence: psychosocial factors in adolescent decision making. Law Hum Behav 1996; 20: 249-272.

35 Richards FH: Maturity of judgement in decision making for predictive testing for nontreatable adult-onset neurogenetic conditions: a case against predictive testing of minors. Clin Genet 2006; 70: 396-401. 